PREPARED FOR THE U.S. DEPARTMENT OF ENERGY, UNDER CONTRACT DE-AC02-76CH03073

PPPL-3831

PPPL-3831

UC-70

Cross-calibrating Spatial Positions

of Light-viewing Diagnostics using Plasma Edge Sweeps in DIII-D

by

W.M. Solomon, K.H. Burrell, P. Gohil, R. Groebner, and D. Kaplan

July 2003

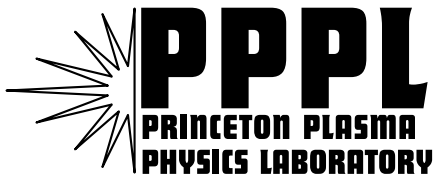

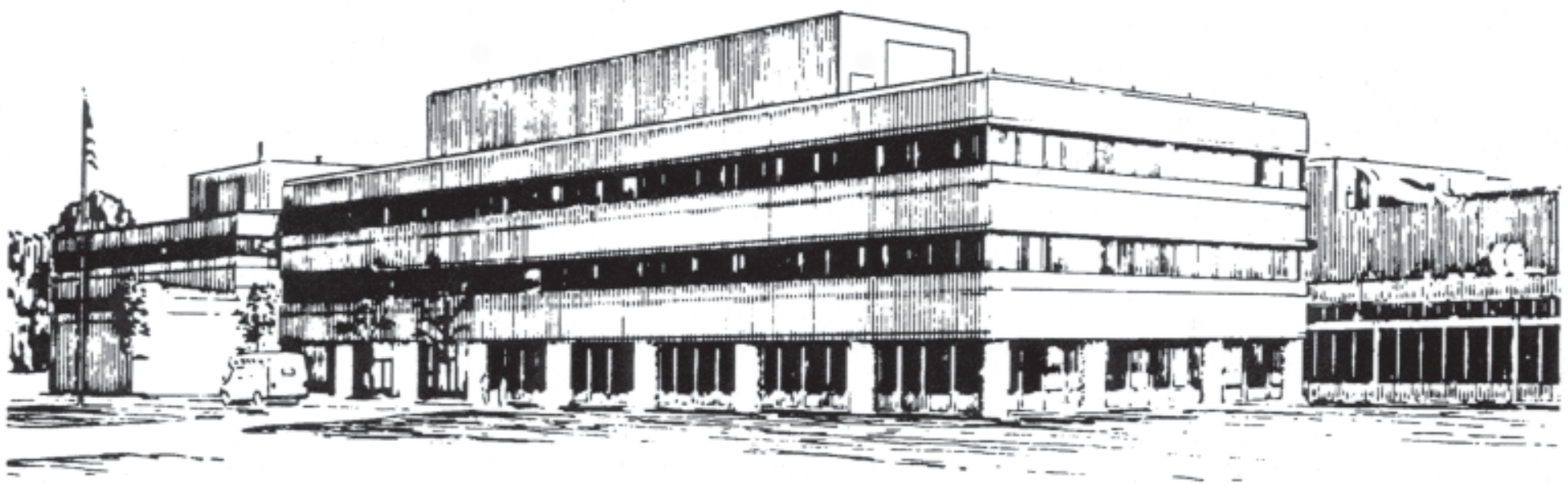

PRINCETON PLASMA PHYSICS LABORATORY PRINCETON UNIVERSITY, PRINCETON, NEW JERSEY 


\section{PPPL Reports Disclaimer}

This report was prepared as an account of work sponsored by an agency of the United States Government. Neither the United States Government nor any agency thereof, nor any of their employees, makes any warranty, express or implied, or assumes any legal liability or responsibility for the accuracy, completeness, or usefulness of any information, apparatus, product, or process disclosed, or represents that its use would not infringe privately owned rights. Reference herein to any specific commercial product, process, or service by trade name, trademark, manufacturer, or otherwise, does not necessarily constitute or imply its endorsement, recommendation, or favoring by the United States Government or any agency thereof. The views and opinions of authors expressed herein do not necessarily state or reflect those of the United States Government or any agency thereof.

\section{Availability}

This report is posted on the U.S. Department of Energy's Princeton Plasma Physics Laboratory Publications and Reports web site in Fiscal Year 2003. The home page for PPPL Reports and Publications is: http://www.pppl.gov/pub_report/

DOE and DOE Contractors can obtain copies of this report from:

U.S. Department of Energy

Office of Scientific and Technical Information

DOE Technical Information Services (DTIS)

P.O. Box 62

Oak Ridge, TN 37831

Telephone: (865) 576-8401

Fax: (865) 576-5728

Email: reports@adonis.osti.gov

This report is available to the general public from:

National Technical Information Service

U.S. Department of Commerce

5285 Port Royal Road

Springfield, VA 22161

Telephone: $1-800-553-6847$ or

(703) $605-6000$

Fax: (703) 321-8547

Internet: http://www.ntis.gov/ordering.htm 


\title{
Cross-calibrating spatial positions of light-viewing diagnostics using plasma edge sweeps in DIII-D
}

\author{
W. M. Solomon* \\ Princeton Plasma Physics Laboratory, Princeton University, NJ 08543 \\ K. H. Burrell, P. Gohil, R. Groebner, and D. Kaplan \\ General Atomics, P.O. Box 85608, San Diego, California 92186-5608
}

(Dated: June 25, 2003)

\begin{abstract}
An experimental technique is presented that permits diagnostics viewing light from the plasma edge to be spatially calibrated relative to one another. By sweeping the plasma edge, each chord of each diagnostic sweeps out a portion of the light emission profile. A non-linear least-squares fit to such data provides superior cross-calibration of diagnostics located at different toroidal locations compared with simple surveying. Another advantage of the technique is that it can be used to monitor the position of viewing chords during an experimental campaign to ensure that alignment does not change over time. Moverover, should such a change occur, the data can still be crosscalibrated and its usefulness retained.
\end{abstract}

*Electronic address: wsolomon@pppl.gov 


\section{INTRODUCTION}

To build an accurate description of a tokamak plasma, it is generally necessary to combine several diagnostic measurements together. An important prerequisite for doing this is that the spatial positions of the measurements are known. Generally, in situ measurements of the spatial positions of each diagnostic are relied upon. However, for detailed H-mode studies of the plasma edge, where gradient scale lengths in DIII-D are of the order of $1 \mathrm{~cm}$, the accuracy attained from these surveying measurements may not be sufficient for diagnostics positioned at different toroidal locations. This is particularly the case on DIII-D, where the machine vessel center is shifted (about $5 \mathrm{~mm}$ ) with respect to the magnetic field. In addition, knowledge of where the neutral beams are located is not accurate to the centimeter level. Cross-calibration is important for the three diagnostics considered here, namely the charge exchange recombination (CER) [1-3], beam emission spectroscopy (BES) [4], and the motional stark effect (MSE) [5, 6], which view light emitted by the neutral beams.

Aside from surveying during machine vents, it may also be necessary for a diagnostic to be re-calibrated during an experimental campaign, since, for example, components may move during operation. A key motivation for this work was to check the location of the BES diagnostic. Data was acquired during the 2001 campaign in quiescent H-mode (QHmode) [7] to determine the radial profile of the density fluctuation associated with the edge harmonic oscillation (EHO). The data indicated that the oscillation was peaked slightly outside the separatrix. In order to verify that this conclusion was valid, it was desirable to check the precise location of the BES chords. One additional complication specific to the BES system is that it uses a stepper motor that allows the chords to be scanned radially on a shot-to-shot basis. Such a scanning system can be prone to additional spatial calibration errors due to the reproducibility of the motorized mount positions.

In DIII-D, the CER, BES, and MSE diagnostics are all capable of collecting light from the plasma edge, and it is possible to tune all three to look at the same $\mathrm{D}_{\alpha}$ wavelength simultaneously. Each chord collects light from the intersection volume between the chord's sightline and the neutral beam. Despite the neutral beam's full width half maximum being about $15 \mathrm{~cm}$ in the horizontal direction, good radial resolution $(\sim<1 \mathrm{~cm})$ is attainable by choosing sightlines essentially tangential to the magnetic flux surface.

Figure 1 shows a plan view of DIII-D, indicating the toroidal locations of these three 
diagnostic, and their associated neutral beams. In particular, note that the CER and BES diagnostics are located almost $5 \mathrm{~m}$ from each other.

In Sec. II, an experimental technique is described that allows the collection of data suitable for cross-calibrating the three diagnostics. Sec. III details the fitting procedure used to determine the relative positions of the diagnostics. Results from this analysis on data taken during the 2001 and 2002 campaigns is presented in Sec. IV.

\section{EXPERIMENTAL TECHNIQUE}

In principle, all that is required to do a rudimentary cross-calibration of the spatial positions of the edge diagnostics is to monitor the same spectral line and independently construct an edge emission profile for each system. Then, provided there is a spatial overlap of the channels from one diagnostic to another, and that the emission profile is described by something more than a simple linear function, the relative locations of the diagnostics can be determined by fitting (or overlaying) the measured profiles. There are, however, limitations to this technique:

1. The quality of the fit depends on the amount of spatial overlap between the chords of different diagnostics;

2. Each chord can produce only one data point on the emission profile;

3. The relative positions of each diagnostic's own chords must be precisely known.

These issues can be largely alleviated by performing an "edge-sweep" of the plasma. During such an edge-sweep, the location of the outboard midplane separatrix $R_{\text {midout }}$ is swept outwards in major radius by adjusting the vertical field. The edge sweep technique was developed specifically to allow the statically located BES chords to map out the location of the EHO. A plot of $R_{\text {midout }}$ during such an edge-sweep is shown in Fig. 2. In this way, a single chord is able to produce many data points along the emission profile, and provided that the sweep is large enough, each chord on each diagnostic can be calibrated independently.

In order for the edge sweep to be useful, we require a quiescent, essentially stationary plasma. Without a quiescent plasma, the fluctuation levels in the density (and hence the light emission) could significantly distort the measured emission profile, particularly in the 
regions where the profile is non-linear. Time traces of the line averaged density, pedestal density, and $\mathrm{D}_{\alpha}$ emission is shown for a typical QH-mode in Fig. 3. Due to the lack of activity on the $\mathrm{D}_{\alpha}$ trace, the QH-mode on DIII-D is an ideal candidate for performing edge sweeps.

For edge sweep cross-calibration, the three diagnostics view the Doppler-shifted $\mathrm{D}_{\alpha}$ line (corresponding to the transition near $656 \mathrm{~nm}$ ) emitted by fast $\mathrm{D}^{0}$ from the neutral beams. The CER system uses Czerny-Turner spectrometers to tune to wavelengths in the range of about $300 \mathrm{~nm}$ to $800 \mathrm{~nm}$ and usually is used to measure the lineshape of the light radiated from charge exchange between neutrals in the neutral beam and low $Z$ impurities. The BES system uses filters to isolate the region near the Doppler-shifted $\mathrm{D}_{\alpha}$ and measures the light intensity. Fluctuations in the light intensity are related back to fluctuations in the local density through atomic physics. The MSE relies on the motional $E=v \times B$ electric field splitting of the $\mathrm{D}_{\alpha}$ line into orthogonally polarized components $(\sigma, \pi)$. MSE measures the polarization of the $\sigma$ component, which allows the local electric field pitch angle to be determined. This is related to both the magnetic field pitch angle $B_{z} / B_{\phi}$, and the radial electric field $E_{r}$. For cross-calibration, we ignore the polarization information and simply use the measured amplitudes.

Figure 4 shows the light intensity of the Doppler-shifted $\mathrm{D}_{\alpha}$, as measured by an edge CER channel during a DIII-D edge sweep. Using output from EFIT [8, 9] magnetic equilibrium analysis, which can give $R_{\text {midout }}$ as a function of time, the time axis can readily be converted to a spatial axis, $R-R_{\text {midout }}$, where $R$ is the position of the chord, as shown in Fig. 5 . During the edge sweep, the neutral beams viewed by the chords to be cross-calibrated are modulated (while keeping the total neutral beam power constant by modulating the other neutral beams out of phase). This allows one to perform background subtraction from the signal, ensuring that the the signal comes from one spatial region in the plasma, rather than along the whole chord. It is worth noting that the background level is observed to drift during the sweep. Using the plasma edge sweep, each chord traces out some segment of the emission profile. Combined with multiple chords, a fairly well-described curve can be generated to represent the emission profile. 


\section{FITTING PROCEDURE}

A code has been written to fit the data from the various diagnostic channels. The positions of the CER channels are assumed known, and the other diagnostics are calibrated relative to the CER system. The data is fit using a non-linear least squares fit. In particular, an emission profile is constructed, which corresponds with the standard function (TANHFIT) used on DIII-D to fit edge profiles [10]. It has the form

$$
\begin{aligned}
I & =A \frac{(1+\alpha x) \mathrm{e}^{-x}-\mathrm{e}^{x}}{\mathrm{e}^{-x}+\mathrm{e}^{x}}+B \\
& =-A \tanh (x)-\frac{A \alpha x}{2} \mathrm{e}^{-x} \operatorname{sech}(x)+B,
\end{aligned}
$$

where

$$
x=\frac{R-R_{\text {midout }}-R_{\text {sym }}}{w},
$$

$R$ is the position of the chord (major radius), and $A, B, \alpha, w$, and $R_{\text {sym }}$ are parameters used to define the emission profile. $R$ is the nominal position of the chord $R_{0}$, plus any offset correction $R=R_{0}+\Delta R$. The offset is a parameter that can either be fitted for each individual chord on the BES or MSE systems, or a single offset may be used to describe a global position shift of a diagnostic. In addition, the absolute intensities $A_{n}$ are not necessarily calibrated from one chord to the next or from one diagnostic to the next. Hence, each chord in the fit (except the first, which can be scaled arbitrarily) needs an additional amplitude parameter. Since the code outputs the scaling amplitudes for each channel as well as the spatial offset quantity, it is therefore also possible to use the edge-sweep technique in order to intensity calibrate chords.

In fact, it is with the additional degrees of freedom given by unscaled intensities that the fitter can no longer reliably cross-calibrate individual channels (i. e. having one $\Delta R$ per chord), since in the (essentially) linear region of the emission profile, having both an amplitude and position parameter guarantees that there is no unique solution. This problem can be overcome with edge sweeps that take a viewing chord from the "top" to the "bottom" of the hyperbolic tangent modeled for the emission profile. This ensures that a chord samples a non-linear region of the profile. While such a large sweep on DIII-D is possible, to date, it has not been attempted. Instead we rely on the relative chord-to-chord spatial calibrations

of each diagnostic to be accurate, and only fit a single $\Delta R$ to describe a global offset of one diagnostic relative to another. 
In total, for $n$ viewing chords (labeled $0,1,2, \ldots, n)$, there are $5+n$ parameters $(A, B$, $\left.\alpha, w, R_{\text {sym }}, \Delta R, A_{1}, A_{2}, \ldots, A_{n}\right)$ that must be fitted. Nonetheless, for a plasma edge sweep performed over a one second period, with neutral beam modulation of $40 \mathrm{~ms}$ on, $40 \mathrm{~ms}$ off, there are more than 10 data points per chord used in the fit. Typically, one can use about 8 CER channels plus any chords from BES or MSE to cross-calibrate. Therefore, a fairly well-constrained non-linear least squares fit can be performed on the data to determine the fitting parameters, the most important for the cross-calibration being $\Delta R$.

The code uses the Levenberg-Marquardt algorithm [11], which is a general non-linear downhill minimization algorithm. It is a dynamic mix between the Gauss-Newton and steepest-descent techniques. The weighted squared difference is computed for each time point in the sweep (corresponding to differing values of $R_{\text {midout }}$ ). In other words, for each data point, the deviate is computed

$$
\delta=\frac{I_{\text {meas }}-I\left(A, B, \alpha, w, R_{\text {sym }}, \Delta R, A_{1}, A_{2}, \ldots, A_{n}\right)}{\sigma}
$$

where $I_{\text {meas }}$ is the measured intensity, and $\sigma$ is standard deviation uncertainty in the measurement. Then,

$$
\chi^{2}=\sum \delta^{2}
$$

is the quantity that is minimized by adjusting the fitting parameters.

\section{RESULTS}

Edge sweeps have been performed on DIII-D during the 2001 and 2002 campaigns. Figure 6 shows the scaled intensities of a set of CER chords and a single BES chord (the fitter was run with constrained $\Delta R=0$ ). It is apparent that there was a significant offset between where the two diagnostics were supposedly viewing. When the code is allowed to adjust the position of the BES chords, a very good fit can be found to the data (Fig. 7). From this fit, the BES chords were determined to be $33.6 \pm 0.9 \mathrm{~mm}$ radially displaced from its nominal position. The error estimates come from the computed covariance matrix, and depend on the input uncertainties in the measurements (the $\sigma$ 's in Eq. (3)). In this study, the uncertainties in the experimentally measured signals are determined empirically (although direct calculation of the errors can in principle be determined from photon statistics, read noise etc.). Specifically, the errors in the measurements are estimated such that the uncertainty 
found in the fitted quantites is representative of the reproducibility obtained by using different time windows for the analysis and different discharges. It should be noted that the millimeter precision achieved is better than the typical spot size of the chords (for CER, the spot size is about $5 \mathrm{~mm}$, for BES it is $\sim 8 \mathrm{~mm}$ ). This is possible due to the large number of data points acquired along the profile by using the edge sweep. Importantly, the spot sizes are still less than the gradient scale lengths, otherwise the interpretation might be more complicated. Also note that any systematic error in $R_{\text {midout }}$ does not affect the value for $\Delta R$, since $R_{\text {midout }}$ is merely used as an arbitrary point of reference. The relative uncertainty in $R_{\text {midout }}$, however, can in principle propagate into both the error and the absolute value obtained for $\Delta R$. Using a Monte Carlo technique, the values for $R_{\text {midout }}$ are perturbed by random amounts (determined from $\delta_{R_{\text {midout }}}$, the error in $R_{\text {midout }}$ ). The uncertainty in $R_{\text {midout }}$ from the magnetic equilibrium reconstruction is estimated to be about $3 \mathrm{~mm}$. Using this value for $\delta_{R_{\text {midout }}}$ contributes an additional $\sim 0.4 \mathrm{~mm}$ to the uncertainty in $\Delta R$.

It was later discovered that the glue holding an alignment mirror on the BES system had deteriorated from the time that the BES was calibrated, and the mirror had slipped. Hence the alignment that was performed on the BES before the campaign was invalid. Nonetheless, the present technique allows the data to be used, since the true viewing position of the BES chords is recovered, assuming the CER did not also shift. The CER system has a rigid alignment, and post-calibration is used to verify that the system does not move during an experimental campaign. Surveying of the BES chords via an in-vessel spatial calibration after the campaign ended confirmed that the offset was about $3 \mathrm{~cm}$.

In comparison, for the same shot, the nominal MSE chords agreed with the CER chord locations to within $<1 \mathrm{~cm}$ of the CER chords (see Fig. 8). In 2002, after the problem with the BES mirror had been corrected, the discrepancy between the CER and BES chords is similar to that of MSE (see Fig. 9).

We now return to the original question - where is the density oscillation associated with the EHO spatially located? Having more precisely determined the position of the BES chords, we may map out the density fluctuation level (more precisely the Doppler-shifted $\mathrm{D}_{\alpha}$ fluctuation level) as a function of space for the dominant EHO mode. The BES has a $1 \mathrm{MHz}$ sampling rate and this higher temporal resolution (compared with either CER or MSE) is necessary to resolve the $\sim 10 \mathrm{kHz}$ EHO in DIII-D. In Fig. 10 (a), the gradient of the fitted Doppler-shifted $\mathrm{D}_{\alpha}$ intensity profile from Fig. 7 is plotted. If the EHO moves a 
rigid density profile back and forth in space, then one would expect that the peak in the fluctuation level measured by BES should correspond with the maximum in this derivative. In Fig. 10 (b), the fluctuation level as measured by the BES channels during an edge sweep with an EHO present is shown, with the chords' spatial positions corrected by the amount output from the cross-calibration $(\sim 34 \mathrm{~mm})$. The fluctuation amplitude is in arbitrary units (normalized to the largest fluctuation level). The peak in the fluctuation does not correspond with the position of the maximum gradient. In fact, the peak measured by the $\mathrm{BES}$ is a little more than $1 \mathrm{~cm}$ further inside the plasma, and so one must concede that the simple, intuitive model of the EHO cannot explain the observations in the Doppler-shifted $\mathrm{D}_{\alpha}$ fluctuations. Since we are comparing the gradient of the $\mathrm{D}_{\alpha}$ emission profile with the fluctuation level in the $\mathrm{D}_{\alpha}$, we do not need to worry about subtleties in the measurement (for example, the inward shift of the profile caused by the time-of-flight before emission).

For comparison, the profile of the fluctuation level obtained from the 2002 BES data (again with the chord spatial positions corrected using the cross-calibration technique) is presented in Fig. 11. Overplotted is the data from 2001 (as per Fig. 7 (b)). In this case, the 2002 fluctuation levels are offset vertically by 0.5 to distinguish the two data sets. One can see that the position of the maximum fluctuation level for the two (quite similar) discharges is quite robust and remains more than $1 \mathrm{~cm}$ further in than the position of steepest gradient. The fact that two quite different configurations of the BES chords (one unintentional!) reproduce the same position for the maximal fluctuations after spatial crosscalibration further adds weight to the precision of the technique.

In summary, the technique of cross-correlating diagnostics viewing light collected from the plasma edge can be immensely useful. By performing an edge sweep of the plasma, it is possible to verify the relative locations of different diagnostics, and achieve superior cross-calibration of these diagnostics located at different toroidal locations than possible with surveying. Indeed, this improved precision is useful for edge QH-mode studies, where the edge gradients are large. Such cross-calibration allows for precise determination of the location of the EHO density fluctuation in the QH-mode plasma. The technique can also serve as a monitor of a diagnostic's components, ensuring that they have not shifted since their last calibration. This gives the experimenter the opportunity to correct any problems before critical data is taken. Furthermore, even after the data has been acquired, as was the case with the BES data in 2001, this technique allows the data to be salvaged by quantifying 
the change to the system.

\section{Acknowledgments}

This work is supported by the U. S. Department of Energy under contracts DE-AC0276CH03073 and DE-AC03-99ER54463. The authors would like to thank R. Nazikian for support of the work, G. McKee for providing the BES data, and R. Jayakumar and M. Makowski for the MSE data.

[1] R. P. Seraydarian, K. H. Burrell, and R. J. Groebner, Review of Scientific Instruments 59, 1530 (1988).

[2] R. H. Seraydarian and K. H. Burrell, Review of Scientific Instruments 57, 2012 (1986).

[3] K. H. Burrell, D. H. Kaplan, P. Gohil, D. G. Nilson, R. J. Groebner, and D. M. Thomas, Review of Scientific Instruments 72, 1028 (2001).

[4] G. McKee, R. Ashley, R. Durst, R. Fonck, M. Jakubowski, and T. K., Review of Scientific Instruments 70, 913 (1999).

[5] B. W. Rice, D. G. Nilson, and D. Wróblewski, Review of Scientific Instruments 66, 373 (1995).

[6] D. Wróblewski, K. H. Burrell, L. L. Lao, P. Politzer, and W. W. P., Review of Scientific Instruments 61, 3352 (1990).

[7] K. H. Burrell, M. E. Austin, D. P. Brennan, J. C. DeBoo, E. J. Doyle, C. Fenzi, C. Fuchs, P. Gohil, C. M. Greenfield, R. J. Groebner, et al., Physics of Plasma 8, 2153 (2001).

[8] L. Lao, H. St. John, R. Stambaugh, A. Kellman, and W. Pfeiffer, Nuclear Fusion 25, 1611 (1985).

[9] Q. Peng, R. J. Groebner, L. L. Lao, J. Schachter, D. P. Schissel, and M. R. Wade, Review of Scientific Instruments 72, 3277 (2001).

[10] R. J. Groebner, D. R. Baker, K. H. Burrell, T. N. Carlstrom, J. R. Ferron, P. Gohil, L. L. Lao, T. H. Osborne, D. M. Thomas, W. P. West, et al., Nuclear Fusion 41, 1789 (2001).

[11] D. W. Marquardt, Journal of the Society for Industrial and Applied Mathematics 11, 431 (1963). 


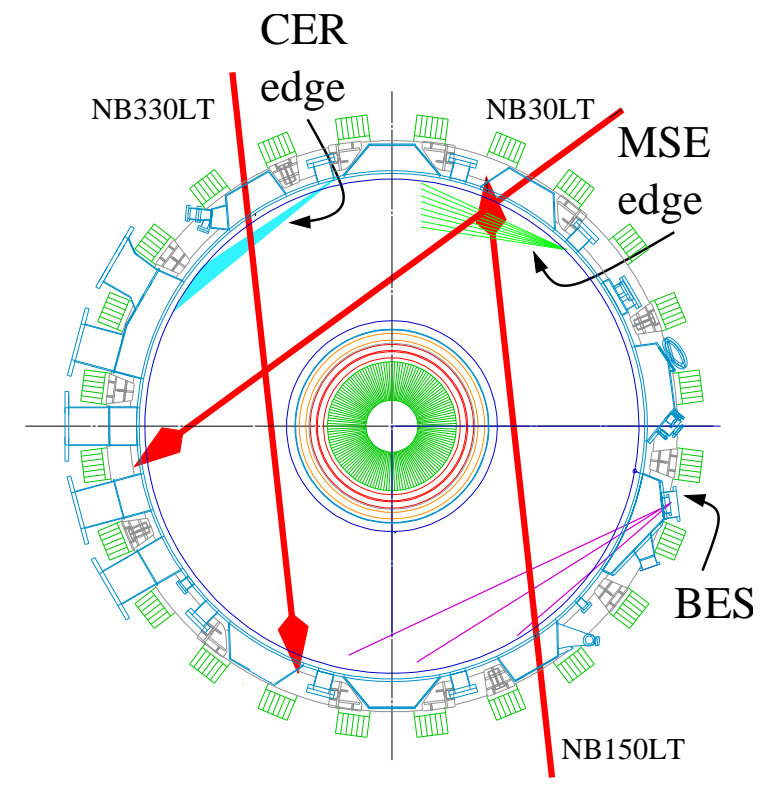

FIG. 1: Plan view of DIII-D showing toroidal locations and views of the CER, BES, and MSE diagnostics. The locations and directions of the three neutral beams viewed by each diagnostic are also indicated. Except for very low densities, the attenuation of the beams in the plasma ensure that the 150LT beam does not reach the MSE diagnostic.

W. M. Solomon 


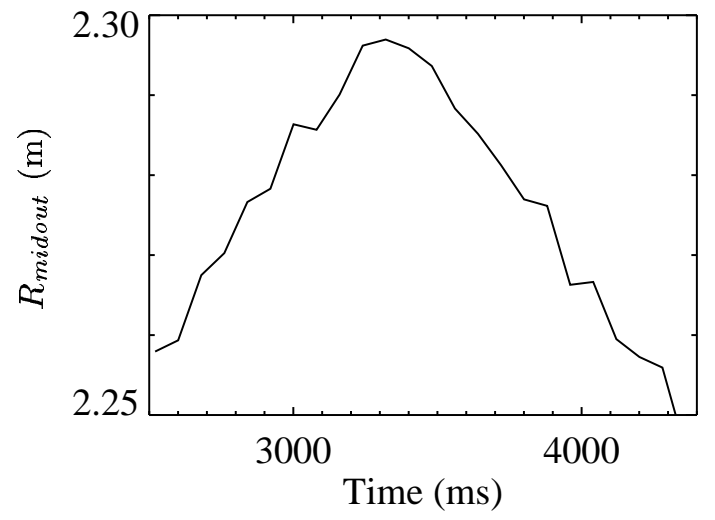

FIG. 2: The location of the outboard midplane separatrix $R_{\text {midout }}$ determined by EFIT as a function of time during a DIII-D edge sweep.

W. M. Solomon 


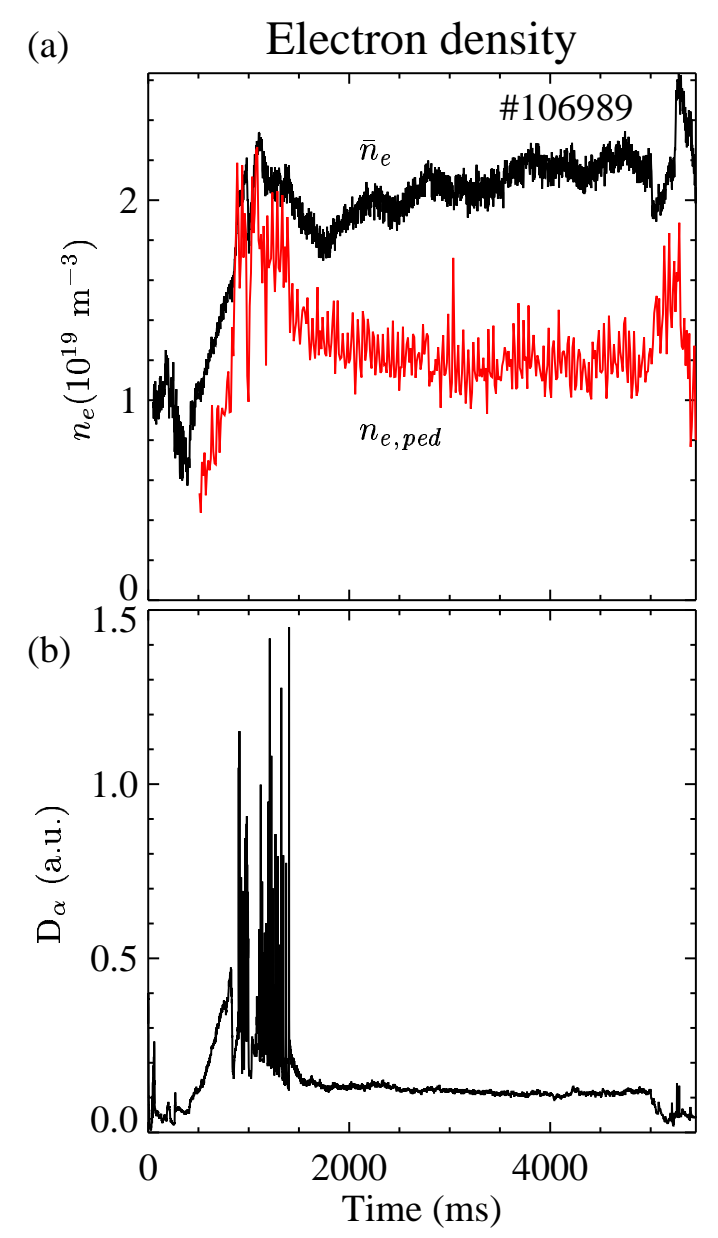

FIG. 3: Time traces of (a) line averaged and pedestal density, and (b) $\mathrm{D}_{\alpha}$ emission during a quiescent H-mode in DIII-D. The lack of fluctuations in $\mathrm{D}_{\alpha}$ indicate quiescent, essentially stationary conditions suitable for the edge-sweep technique.

W. M. Solomon 


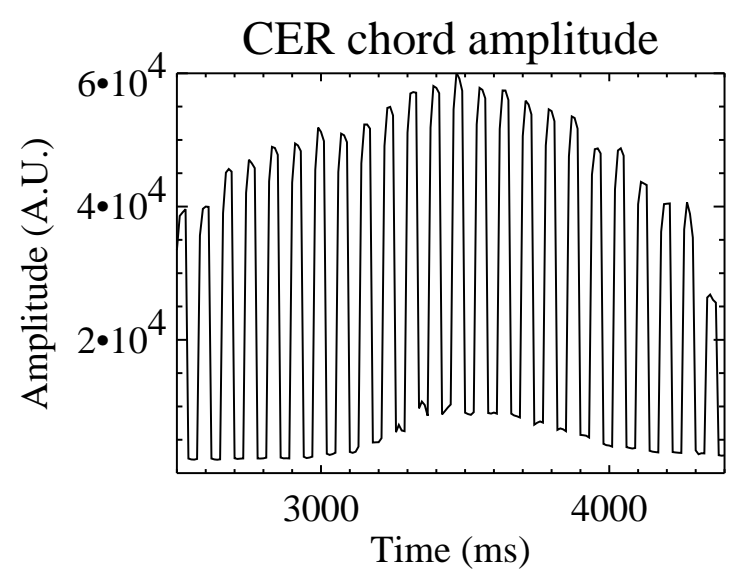

FIG. 4: Data acquired by a single CER channel (T09) during the edge sweep shown in Fig. 2. During this period of time, the neutral beam viewed by this chord is modulated with $40 \mathrm{~ms}$ on, $40 \mathrm{~ms}$ off. The total neutral beam power, however, is kept constant by modulating other neutral beams out of phase with this one. The signal during the off period is subtracted from that taken with the beam on to remove the time-varying background from the plasma.

W. M. Solomon 


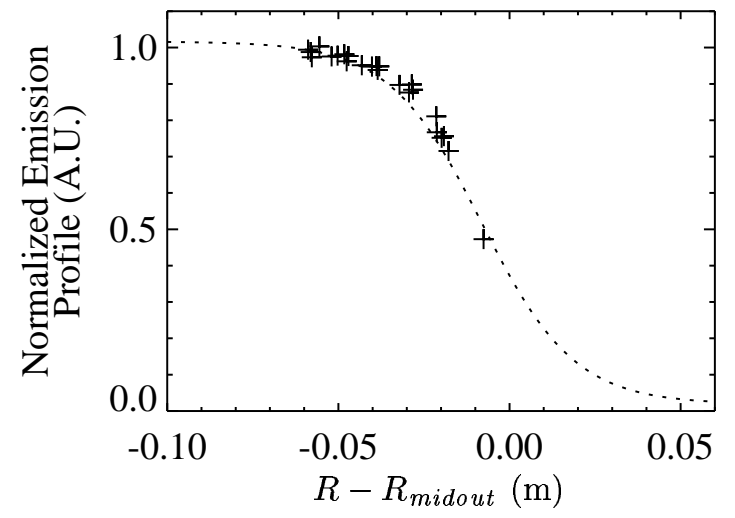

FIG. 5: Data from a single CER chord plotted against its distance from the separatrix, $R-R_{\text {midout }}$. The dashed line is the emission profile determined from all the data fitted to the function in Eq. (1). Each chord traces out a portion of the light emission profile.

W. M. Solomon 


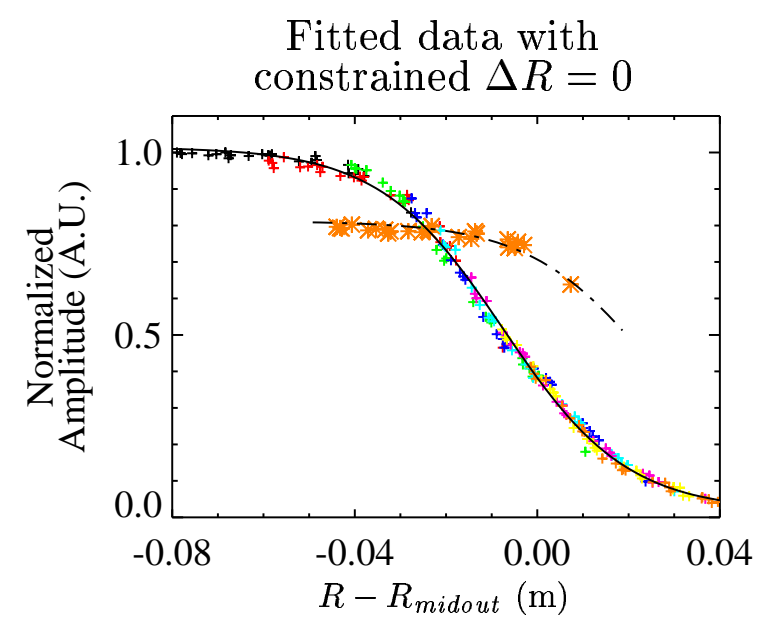

FIG. 6: Non-linear least-squares fitter attempts to fit a single BES channel against an array of CER channels. It is constrained with $\Delta R=0$ (but allowed to scale the intensity). A clear spatial offset is evident.

W. M. Solomon 


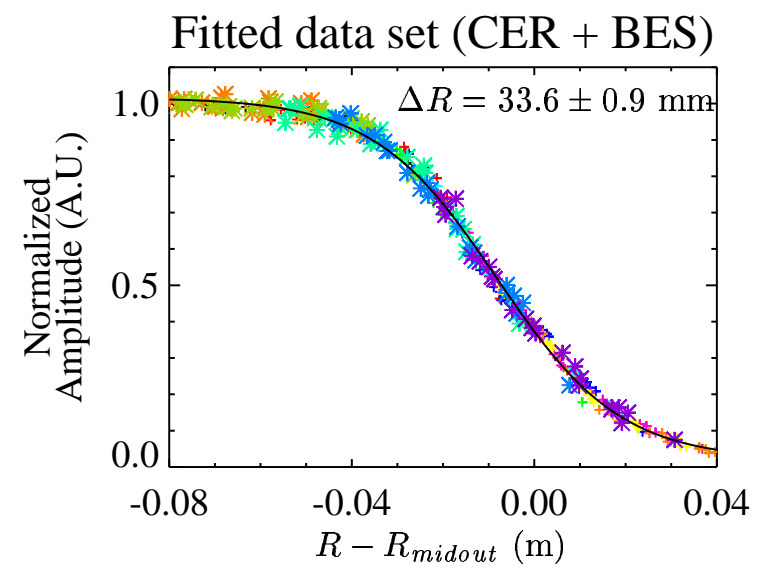

FIG. 7: Multiple CER and BES channels fit to the emission profile. The BES chords were found to be offset relative to the CER chords by more than $30 \mathrm{~mm}$. It was discovered that an alignment mirror on the BES system had shifted during operation.

W. M. Solomon 


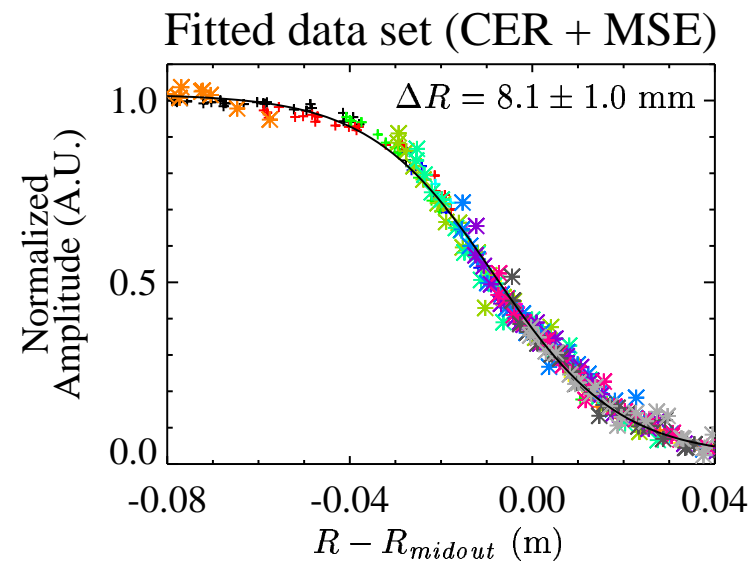

FIG. 8: Multiple CER and MSE channels fit to the emission profile In this case, the relative offset between the two is less than $1 \mathrm{~cm}$, and is consistent with the accuracy that can be achieved with surveying type calibrations at toroidally displaced locations.

W. M. Solomon 


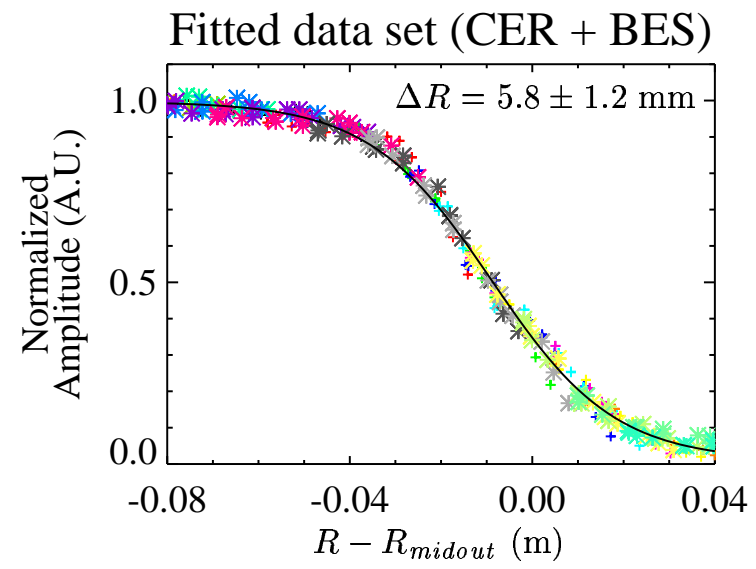

FIG. 9: In comparison with Fig. 7, after the alignment mirror had been corrected, the discrepancy between the CER and BES systems is now within the accuracy of the surveying calibration.

W. M. Solomon 


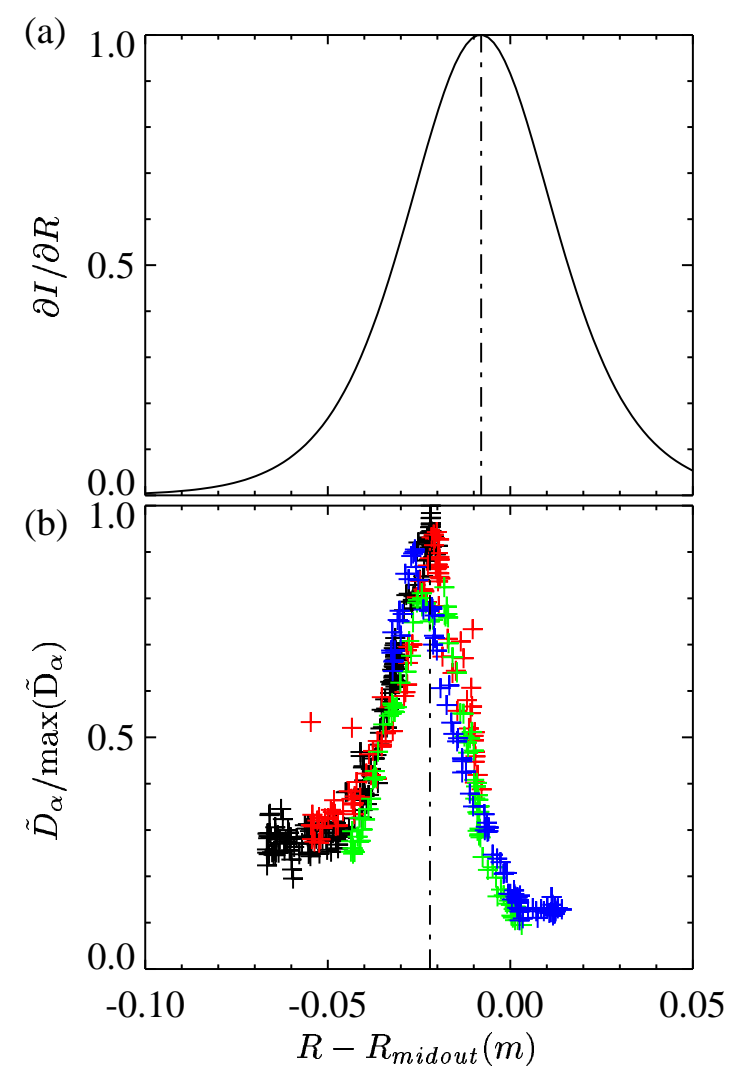

FIG. 10: (a) Normalized gradient of the Doppler-shifted $\mathrm{D}_{\alpha}$ emission profile. (b) BES Dopplershifted $\mathrm{D}_{\alpha}$ fluctuation level profile (normalized to one) for 2001 edge-sweep in the presence of an EHO.

W. M. Solomon 


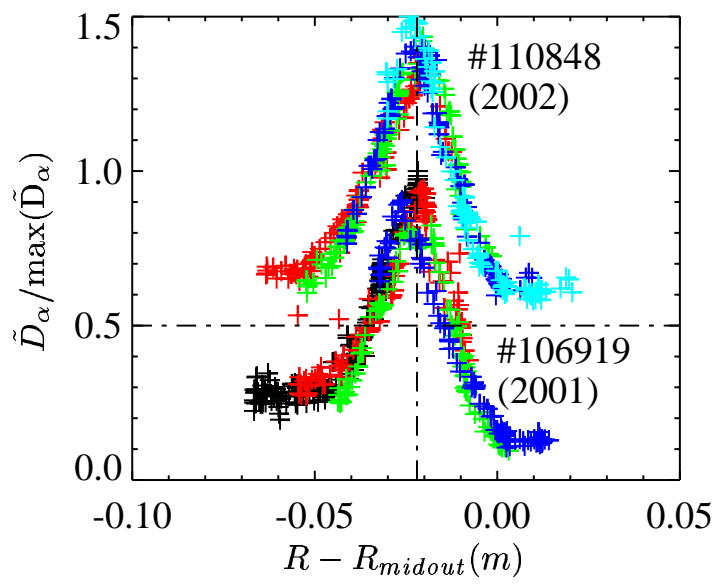

FIG. 11: Overplot of BES Doppler-shifted $\mathrm{D}_{\alpha}$ fluctuation level profile (normalized to one) during edge-sweep in the presence of an EHO for 2001 data (lower curve) and 2002 data (upper curve).

W. M. Solomon 


\section{External Distribution}

Plasma Research Laboratory, Australian National University, Australia

Professor I.R. Jones, Flinders University, Australia

Professor João Canalle, Instituto de Fisica DEQ/IF - UERJ, Brazil

Mr. Gerson O. Ludwig, Instituto Nacional de Pesquisas, Brazil

Dr. P.H. Sakanaka, Instituto Fisica, Brazil

The Librarian, Culham Laboratory, England

Mrs. S.A. Hutchinson, JET Library, England

Professor M.N. Bussac, Ecole Polytechnique, France

Librarian, Max-Planck-Institut für Plasmaphysik, Germany

Jolan Moldvai, Reports Library, Hungarian Academy of Sciences, Central Research Institute for Physics, Hungary

Dr. P. Kaw, Institute for Plasma Research, India

Ms. P.J. Pathak, Librarian, Institute for Plasma Research, India

Ms. Clelia De Palo, Associazione EURATOM-ENEA, Italy

Dr. G. Grosso, Instituto di Fisica del Plasma, Italy

Librarian, Naka Fusion Research Establishment, JAERI, Japan

Library, Laboratory for Complex Energy Processes, Institute for Advanced Study, Kyoto University, Japan

Research Information Center, National Institute for Fusion Science, Japan

Dr. O. Mitarai, Kyushu Tokai University, Japan

Dr. Jiangang Li, Institute of Plasma Physics, Chinese Academy of Sciences, People's Republic of China

Professor Yuping Huo, School of Physical Science and Technology, People's Republic of China

Library, Academia Sinica, Institute of Plasma Physics, People's Republic of China

Librarian, Institute of Physics, Chinese Academy of Sciences, People's Republic of China

Dr. S. Mirnov, TRINITI, Troitsk, Russian Federation, Russia

Dr. V.S. Strelkov, Kurchatov Institute, Russian Federation, Russia

Professor Peter Lukac, Katedra Fyziky Plazmy MFF UK, Mlynska dolina F-2, Komenskeho Univerzita, SK-842 15 Bratislava, Slovakia

Dr. G.S. Lee, Korea Basic Science Institute, South Korea

Institute for Plasma Research, University of Maryland, USA

Librarian, Fusion Energy Division, Oak Ridge National Laboratory, USA

Librarian, Institute of Fusion Studies, University of Texas, USA

Librarian, Magnetic Fusion Program, Lawrence Livermore National Laboratory, USA

Library, General Atomics, USA

Plasma Physics Group, Fusion Energy Research Program, University of California at San Diego, USA

Plasma Physics Library, Columbia University, USA

Alkesh Punjabi, Center for Fusion Research and Training, Hampton University, USA

Dr. W.M. Stacey, Fusion Research Center, Georgia Institute of Technology, USA

Dr. John Willis, U.S. Department of Energy, Office of Fusion Energy Sciences, USA

Mr. Paul H. Wright, Indianapolis, Indiana, USA 
The Princeton Plasma Physics Laboratory is operated by Princeton University under contract with the U.S. Department of Energy.

\author{
Information Services \\ Princeton Plasma Physics Laboratory \\ P.O. Box 451 \\ Princeton, NJ 08543
}

Phone: 609-243-2750

Fax: 609-243-2751

e-mail: pppl_info@pppl.gov

Internet Address: http://www.pppl.gov 